\title{
Energy Efficient Cooperative Coalition Selection in Cluster-based Capillary Networks for CMIMO IoT Systems
}

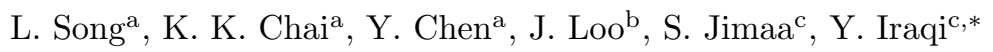 \\ ${ }^{a}$ School Electronic Engineering and Computer Science, Queen Mary University of London, \\ London, United Kingdom \\ ${ }^{b}$ School of Computing and Engineering, University of West London, \\ London, United Kingdom \\ ${ }^{c}$ Electrical and Computer Engineering Department, Khalifa University, \\ Abu Dhabi, United Arab Emirates
}

\begin{abstract}
The Cooperative Multiple-input-multiple-output (CMIMO) scheme has been suggested to extend the lifetime of cluster heads $(\mathrm{CHs})$ in cluster-based capillary networks in Internet of Things (IoT) systems. However, the CMIMO scheme introduces extra energy overhead to cooperative devices and further reduces the lifetime of these devices. In this paper, we first articulate the problem of cooperative coalition's selection for CMIMO scheme to extend the average battery capacity among the whole network, and then propose to apply the quantuminspired particle swarm optimization (QPSO) to select the optimum cooperative coalitions of each hop in the routing path. Simulation results proved that the proposed QPSO-based cooperative coalition's selection scheme could select the optimum cooperative sender and receiver devices in every hop dynamically and outperform the virtual MIMO scheme with a fixed number of cooperative devices.
\end{abstract}

Keywords: IoT systems, cluster, cooperative communication, energy efficiency, QPSO

\section{Introduction}

Internet of Things (IoT) systems are observed to have prospective to enhance the operational efficiency of many industrial applications. [ $\left.R_{2,1}\right]$ Information Handling Services (IHS) anticipates that the IoT market will grow from an established set of 15.4 billion devices in 2015 to about 31 billion devices in 2020 and 75.4 billion in 2025 [1]. The Internet connects people via business and social 
communication or with systems via business transaction such as internet banking or e-commerce. On the other hand, the IoT goal is to connect machines and systems sensors and actuators, in order to collect meaningful information from these systems and take actions to enhance human productivity and efficiency. IoT is driving the proliferation of connected devices from around 15 billion plus today to over 30 billion in 2020. By doing so, IoT takes the meaning of interconnectivity to a whole new level. There is a long list of great benefits that IoT offers. The following are some examples: Smarter homes and offices that can save energy costs, better health care via remote monitoring of patients [2], reminders of mundane tasks such as payment of utility bills and parking meters. Other examples include smart lighting of streets, automatic sensing and control of traffic signals, remote monitoring of assembly line and production system to maximize operational efficiency, reliability and safety in manufacturing facilities, and smart automobiles that can summon assistance if required and assist in controlling vehicle speed based on traffic and environmental conditions.

There is an increasing demand of vast numbers of trustworthy devices provided with short-range radio interfaces, such as Zigbee, low-power Wi-Fi etc. to deliver connectivity to other devices in IoT systems in order to preserve the operational efficiency. Reliable and energy efficient communications for the IoT systems are improved due to the introduction of capillary networks. These networks are specific local networks comprising of a group of wireless devices to be connected to other communication infrastructure such as mobile networks [3]. Such networks use clustering mechanisms to enhance energy efficiency [4].

$\left[R_{1,5}\right]$ In [5] the authors analyses the performance of LEACH-based wireless sensor networks regarding lifetime and throughput. The LEACH protocol is a well-known routing protocol for cluster based wireless sensor networks. The benefit of LEACH is that each node has an equal probability to be a cluster head. In this case, the energy dissipation of each node is relatively fair. In LEACH protocol, time is partitioned into many rounds, in each round, all the nodes try to be $\mathrm{CH}$ according to a predefined criterion. The authors focus on how to choose the time length of each round, to increase the lifetime of the network and improve throughput, which is denoted as the number of data packets conveyed to the sink node. The relationship between the lifetime and throughput to the time length of each round is utilized to improve the performance of cluster-based wireless sensor networks in terms of lifetime and throughput. The reasonable number of frames in a LEACH round is deduced to prolong the lifetime and increase the throughput.

$\left[R_{1,5}\right]$ The authors in [6] investigates energy efficiency in cooperative cellular networks. Based on the double auction theory, they model the optimal relay assignment problem, which aims at improving the performance of cell-edge users (CEUs) with energy efficiency optimization. In the proposed auction-based model, the selfish nature of users is taken into consideration, which means users in the idle state are unwilling to relay the information for active CEUs unless they are paid enough. Therefore, they use mark-up to determine the bid and ask. Furthermore, the energy efficiency (EE) is defined and the model for optimizing the EE is built. An energy-efficient maximum weighted matching 
algorithm (EE-MWM) is proposed to solve the EE optimization problem. Also, the performance of EE-MWM is evaluated in terms of EE, capacity and social welfare, which shows that EE-MWM can greatly improve the performance of cooperative cellular networks. Cooperative communications improve the performance of wireless networks by letting nodes cooperate to obtain spatial diversity gains. To achieve cooperation gains, suitable relay node(s) should be chosen according to network topology and channel state information (CSI). But, in ad hoc wireless networks, the CSI is random, and the network topology is changing. This complicates relay selection, and in turn, makes it hard to perform cooperative communications in ad hoc wireless networks. To address this issue, the authors in [7] propose blind cooperative communications (BCC) for multihop ad hoc wireless networks. In BCC, intermediate nodes and the target node in a multihop path first listen and store the data from two-hop-away upstream nodes, and then merge the stored data with the data forwarded by the upstream to achieve diversity gains. The performance of BCC is assessed by analysis and simulations to confirm that BCC can significantly enhance the packet delivery ratio and the average end-to-end throughput. Compared with existing cooperation methods, BCC needs neither CSI and topology information nor additional signaling overheads, making it very easy to implement in changing multihop ad hoc wireless networks.

$\left[R_{2,3}\right]$ To meet the requirements of high EE and large system capacity for the $5 \mathrm{G}$ Internet IoT, the use of massive multiple-input multiple-output technology has been launched in the massive IoT (mIoT) network, where a large number of devices are connected and scheduled simultaneously. The authors in [8] considers the energy-efficient design of a multipair decode-and-forward relaybased IoT network, in which multiple sources simultaneously transmit their information to the corresponding destinations via a relay equipped with a large array. Furthermore, a simple yet efficient lower bound of the EE is obtained. Based on this, a low-complexity energy-efficient resource allocation strategy of the mIoT network is proposed under the specific quality-of-service constraint. Their proposed strategy determines the near-optimal number of relay antennas, the near-optimal transmit power at the relay, and near-optimal density of active mIoT device pairs in a given coverage area.

$\left[R_{2,3}\right]$ Considering physical sensors with certain sensing capabilities in an IoT sensory environment, the authors in [9] propose an efficient energy management framework to control the duty cycles of these sensors under qualityof-information (QoI) expectations in a multitask-oriented environment. Contrary to past research efforts, their proposal is transparent and compatible both with the underlying low-layer protocols and diverse applications, and preserving energy-efficiency in the long run without sacrificing the QoI levels attained. In particular, they first introduce the novel concept of QoI-aware sensor-to-task relevancy to explicitly consider the sensing capabilities offered by a sensor to the IoT sensory environments, and QoI requirements required by a task. Second, they propose a novel concept of the critical covering set of any given task in selecting the sensors to service a task over time. Third, the energy management decision is made dynamically at runtime, to reach the optimum for long-term 
application arrivals and departures under the constraint of their service delay.

$\left[R_{2,3}\right]$ Simultaneous wireless information and power transfer (SWIPT) is anticipated to have great applications in $5 \mathrm{G}$ communication systems and the Internet of Things. The authors in [10] address the EE optimization problem for SWIPT multiple-input multiple-output broadcast channel (BC) with timeswitching (TS) receiver design. Their aim is to maximize the EE of the system while meeting certain constraints in terms of maximum transmit power and minimum harvested energy per user. The coupling of the optimization variables, namely transmit covariance matrices and TS ratios, leads to an EE problem which is nonconvex, and hence very difficult to solve directly. Hence, they transform the original maximization problem with multiple constraints into a suboptimal min-max problem with a single constraint and multiple auxiliary variables. They propose a dual inner/outer layer resource allocation framework to tackle the problem. For the inner-layer, they invoke an extended SWIPTbased BC-multiple access channel (MAC) duality approach and provide two iterative resource allocation schemes under fixed auxiliary variables for solving the dual MAC problem. A subgradient searching scheme is then proposed for the outer-layer in order to obtain the optimal auxiliary variables. Numerical results confirm the effectiveness of their proposed algorithms and demonstrate that important performance gain in terms of EE can be realized by using the proposed extended BC-MAC duality-based algorithm.

$\left[R_{2,3}\right]$ The authors in [11] present a naming, addressing, and profile server (NAPS) as a middleware to bridge different platforms in IoT sensory environments. Given massive amount of heterogeneous devices deployed across different platforms, NAPS serves as the key module at the back-end data center to aid the efficient upstream sensory data collection, content-based data filtering and matching, and downstream efficient control by applications. While previous research efforts only focus on a specific standard or protocol, they aim to design a middleware component servicing dynamic application needs, and sensors/actuators deployment and configurations across different platforms. Specifically, the authors of this paper propose a complete design of NAPS, including its key functionalities, system flows, interfaces, and individual module design. They further propose a unique device naming and addressing convention, and show its applicability to a few widely-used standards and protocols. They also propose an efficient identifier generation scheme and demonstrate a full implementation of the above designs with a case study, including a service registration portal.

Clustering mechanisms organize the devices into different clusters and choose cluster heads $(\mathrm{CHs})$ that consequently collect data from all cluster members $(\mathrm{CMs})$ and transmit the collected data to the sink node via communication infrastructure networks. However, the CHs consume more energy compared to other devices in the network as they take more responsibility and disperse additional energy to transmit grouped data to the sink node. Cooperative multiple-input-multiple-output (CMIMO) transmission scheme was then proposed to solve the aforementioned problem [12]. CMIMO introduces additional cooperative nodes (Coops) at both sending and receiving ends to help $\mathrm{CH}$ in 
long-haul transmission which is the most energy consuming phase of the communication between the cluster and the sink node. In [13], the authors proposed a novel fair cooperative communication scheme that inspires devices to contribute in cooperative communication by offering extra rewards. A Coop is selected from CMs within the same cluster if two conditions are satisfied: the first is that Signal-to-Noise Ratio (SNR) of the received signal sent by the CM is larger than a predefined SNR threshold level, and the second condition is that the $\mathrm{CM}$ is within the domain of the destination cluster. Via simulation results and numerical analysis, it was demonstrated that the proposed scheme consumes far less energy than partial cooperative and non-cooperative schemes. However, the energy level of devices is not considered in [13]. In [14], a solution for nonuniform energy consumption in the cluster based multi-hop wireless networks through adaptive selection of cooperative MIMO schemes has been proposed by the authors. The authors also have shown that the multi-hop cooperative MIMO in relay clusters result in reduced energy consumption as compared to the non-cooperative case, and the adaptive selection of cooperative transmission delivers uniform energy consumption in all the clusters. However, the Coops selection is not studied in [14].

The above-mentioned challenges raise the concerns to find an optimum set of cooperative coalition's at both transmit and receive sides in cluster-based capillary networks with the objectives of energy efficiency. Evolutionary algorithms are believed to have potential in solving such a coalitions selection problem [15]. Quantum-inspired particle swarm optimization (QPSO), one of the evolutionary algorithms merges the advantages of the quantum computing theory and evolutionary algorithms. Compared with particle swarm optimization (PSO), QPSO embraces novel rotation angle and quantum bit techniques so that it has the characteristics of strong searching capability, short-computing time, rapid convergence, and small-population size [16].

The main contributions of this work are summarized as follows:

- First, we frame the energy efficiency optimization into the optimum cooperative coalition's selection problem with the aim of maximizing the average battery capacity among the whole network. In particular, the energy consumption of each transmission phase is framed in terms of number of transmitters and receivers, packet size as well as a pre-determined bit error rate (BER) threshold. Furthermore, a battery model in [17] is used to evaluate the energy consumption of all devices by battery capacity.

- Second, we propose to select cooperative coalitions at the sender and receiver sides of each hop in the routing path by QPSO. By taking advantage of the low complexity and fast convergence of QPSO, we formulate the possible cooperative coalitions by quantum-coded particles. All particles are hovered through the whole search space by updating the fitness value, i.e. the average battery capacity, until reaching the pre-defined maximum generation.

The rest of this paper is organized as follows: Section II introduces the system model including the problem formulation, the power consumption model, and 
the battery model. In Section III, the QPSO algorithm has been explained in detail and also we propose the use of QPSO to achieve the optimum cooperative coalitions in the routing path with the objective of maximizing the average battery operating time in the network. Simulation results and conclusions are provided in Sections IV and V, respectively.

\section{System Model and Problem Formulation}

In this work the system model, shown in Fig. 1, focuses on one routing path from Cluster 1 to the capillary gateway through $n$ hops. The system model considers a typical energy-limited cluster-based capillary network for IoT systems with $\mathcal{N}_{\text {total }}$ devices. All devices in the routing path have been classified into four classes: CHs, CMs, cooperative sender devices (SCoops), and cooperative receiver devices (RCoops). Denote the number of $\mathrm{CHs}$ in the network to be $\mathcal{N}_{C H}$, which also indicates the number of clusters in the network, while the number of CMs in cluster $i$ to be $\mathcal{N}_{C M}(i)$ where $i \in\left[1, \cdots, \mathcal{N}_{C H}\right]$. Also the number of SCoop and RCoop in cluster $i$ are denoted $\mathcal{N}_{S C o o p}(i)$ and $\mathcal{N}_{R C o o p}(i)$ respectively, where $i \in\left[1, \cdots, \mathcal{N}_{C H}\right]$.

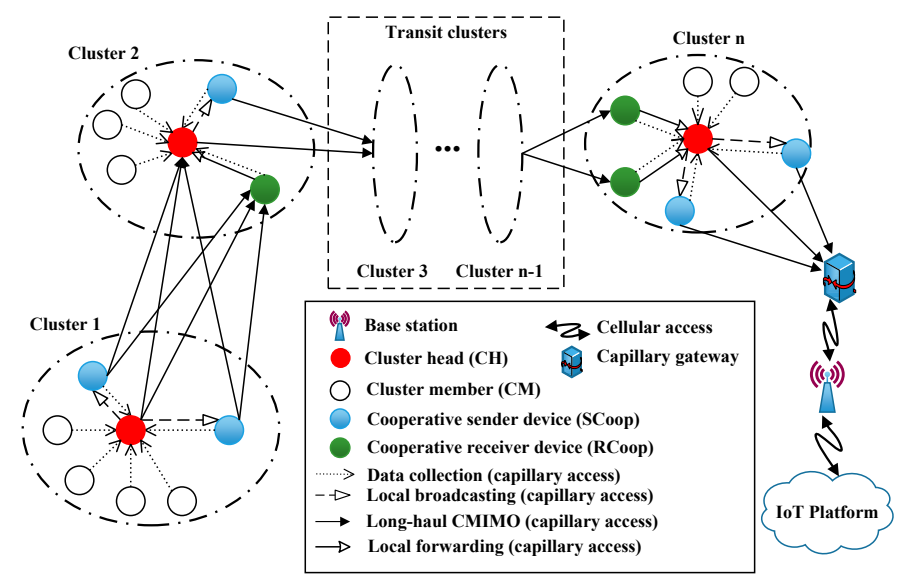

Figure 1: System model.

In this paper, we adopt the additive white Gaussian noise (AWGN) channel with squared power path loss for intracluster communications within the cluster, and the frequency nonselective and slow Rayleigh fading channel for long-haul transmission in each hop. Moreover, since the communications environment is more complex in the long-haul transmission, we assume that the long-haul transmission with respect to the wavelength gives rise to independent fading coefficients. In developing the strategy, $M$-ary Quadrature Amplitude Modulation (MQAM) is adopted. Furthermore, we assume the energy level of capillary gateway is infinite, therefore, the energy consumption of capillary gateway is omitted. 
In Fig. 1, considering the long range scenario, the data flow from Cluster 1, i.e. the source cluster to the capillary gateway, is through several transit clusters by CMIMO scheme to reduce the long-haul transmission overhead. In particular, $\mathrm{CH}$ in cluster 1 at the transmission side liaises with its SCoops to send data to their neighboring cluster, i.e. Cluster 2, by forming a virtual multi-antenna diversity system. Concurrently, the $\mathrm{CH}$ in Cluster 2 liaises with its RCoops to receive the MIMO modulated data from Cluster 1 in the reception side. In this way, all the transit clusters from Cluster 2 to the capillary gateway not only forward their own data but also relay the MIMO modulated data from their neighboring clusters in the routing path.

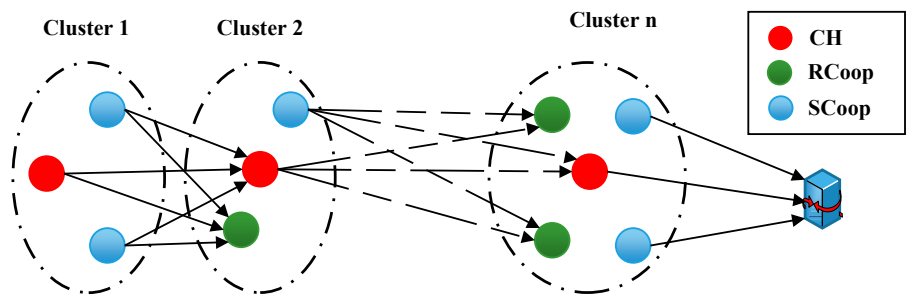

Figure 2: Inter-cluster communication

The transmission process of every cluster in the routing path consists of the following phases:

- Data collection (DC) phase. As shown in Fig. 3a, CH collects and aggregates data from all other non-CH devices.

- Local broadcasting (LB) phase. As shown in Fig. 3b, CH broadcasts the aggregated data to all SCoops at the transmission side.

- Long-haul cooperative transmission (LH) phase. As shown in Fig. 2, CH and its SCoops in the transmission cluster jointly encode and send the aggregated data to the $\mathrm{CH}$ and its RCoops in the adjacent cluster or the capillary gateway based on the cooperative technique investigated in [18] which is such that the

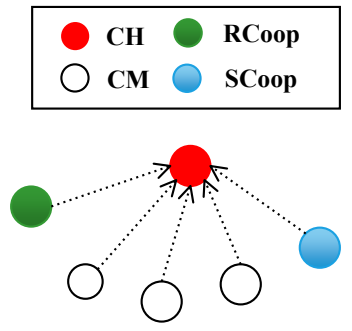

(a) Data collection phase

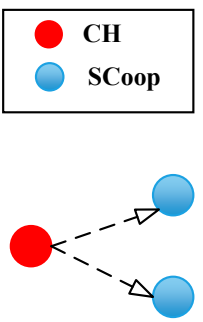

(b) Local broadcasting phase
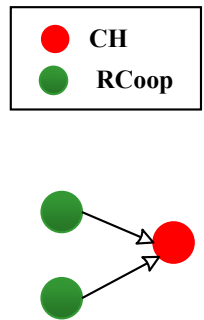

(c) Local forwarding phase

Figure 3: Inter-cluster communication 
cooperative coalitions share their antennas to create a virtual array through distributed transmission and signal processing.

- Local forwarding (LF) phase. In this phase as shown in Fig. 3c, all RCoops transmit the MIMO modulated data to their $\mathrm{CH}$ at the reception side. The MIMO modulated data are obtained from the transmission cluster. Then the reception $\mathrm{CH}$ decodes and combines these data with its cluster data collected in the data collection phase. Finally, the combined data is further directed through the next hop.

In addition, we use the transmission operation in [19], which is divided into several rounds during the time period from the initialization of the network to the last device running out of energy. The transmission is operated in two phases of one round as shown in Fig. 4 set-up phase and steady state phase. During the set-up phase, the gateway executes the clustering algorithm to select $\mathrm{CHs}$ and form clusters, the routing algorithm to construct the routing table, and the cooperative coalition selection algorithm to select SCoops and RCoops in each cluster. The steady state phase consists of several time frames. All the four phases in Fig. 2 and Fig. 3 are performed during one time frame, where time division multiple access (TDMA) scheduling is employed. Express the duration of one time frame to be $t_{\Delta}$ and the number of time frames of the steady state phase in one round to be $\mathcal{N}_{\text {frame }}$. Generally, in comparison with the duration of the steady state phase, the length of set-up phase is much shorter, which can be ignored, hence the duration time of one round is $T_{\text {round }}=t_{\Delta} \times \mathcal{N}_{\text {frame }}$.

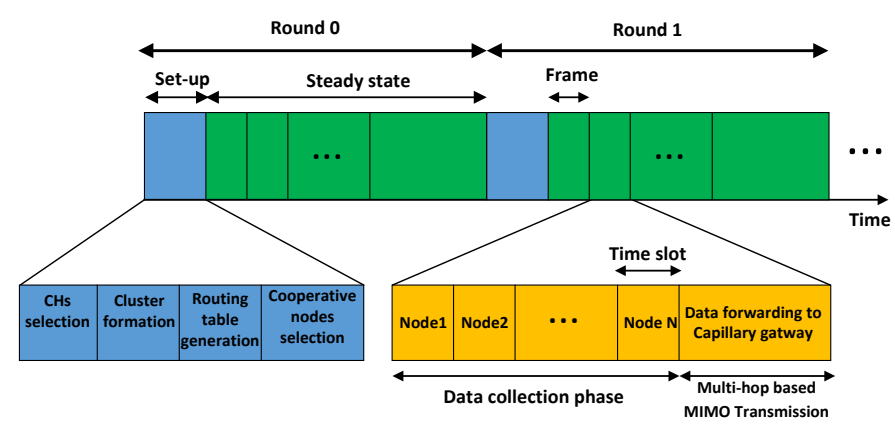

Figure 4: Transmission structure in cluster-based IoT system

\subsection{Battery Model}

In this work, the battery model in [17] is used. When a battery discharges at current rate $I_{c}$ in time period $\left[t_{s}, t_{e}\right]$, the available capacity $C_{a v l}\left(I_{c}, T_{o p}, t_{s}, t_{e}, \beta^{2}\right)$ can be expressed as follows,

$$
C_{a v l}\left(I_{c}, T_{o p}, t_{s}, t_{e}, \beta^{2}\right)=C_{i n i t}-I_{c} F\left(T_{o p}, t_{s}, t_{e}, \beta^{2}\right),
$$


where

$$
\begin{aligned}
& F\left(T_{o p}, t_{s}, t_{e}, \beta^{2}\right)=\left(t_{e}-t_{s}\right) \\
& +2 \sum_{k=1}^{\infty} \frac{e^{-\beta^{2} k^{2}\left(T_{o p}-t_{e}\right)}-e^{-\beta^{2} k^{2}\left(T_{o p}-t_{s}\right)}}{\beta^{2} k^{2}},
\end{aligned}
$$

$C_{\text {init }}$ is the initial battery capacity, $\beta^{2}$ is a constant related to the diffusion rate of the battery, which can be determined by data fitting [20], and $T_{o p}$ is the total operating time of the battery. In (2) there are two terms, the first term $I_{c}\left(t_{e}-t_{s}\right)$ is the consumed capacity by the given current load $I_{c}$, and the second term is the capacity loss due to the current effect.

\subsection{Energy Consumption Model}

The power consumption model defined in [21] is used in this work. The total power consumption alongside a single path can be split into two main components: one is the power consumption of all power amplifiers $P_{P A}$ and the other component is the power consumption of all other circuit blocks $P_{c}$. Note that the power consumption of power amplifiers is linearly dependant on the transmit power $P_{t}$, as in [21]. Then the power consumption per link can be expressed as

$$
P=P_{P A}+P_{c}=(1+\alpha) P_{t}+P_{c}
$$

where $\alpha=\xi / \eta-1$ with $\eta$ being the drain efficiency of the RF power amplifier, and $\xi$ being the peak-to-average ratio, which is dependent on the modulation scheme and the associated constellation size [22]. As referred to [21], $\xi=3(M-$ $2 \sqrt{M}+1) /(M-1)$ in $M$ QAM coded communication.

As discussed in [21], denote the number of transmitters and receivers to be $\mathcal{N}_{t}$ and $\mathcal{N}_{r}$ respectively, we estimate the circuit power consumption $P_{c}$ as

$$
\begin{aligned}
& P_{c} \approx \mathcal{N}_{t}\left(P_{D A C}+P_{\text {mix }}+P_{f i l t}\right)+2 P_{\text {syn }} \\
& +\mathcal{N}_{r}\left(P_{L N A}+P_{\text {mix }}+P_{I F A}+P_{f i l r}+P_{A D C}\right),
\end{aligned}
$$

where $P_{D A C}, P_{m i x}, P_{f i l t}, P_{s y n}, P_{L N A}, P_{I F A}, P_{f i l r}$ and $P_{A D C}$ are the power consumption values of the $\mathrm{D} / \mathrm{A}$ converter, the mixer, the active filters at the transmitter side, the frequency synthesizer, the low noise amplifier, the intermediate frequency amplifier, the active filters and the A/D converter at the receiver side, respectively. $P_{c}$ comprises the transmitting circuit power $P_{c t}$ and the receiving circuit power $P_{c r}$.

For a general communication link, the energy consumption per bit can be formulated as

$$
E_{b t}=\frac{(1+\alpha) P_{t}+P_{c}}{R_{b}},
$$

where $R_{b}=B \log _{2} M$ is the bit rate for $M Q A M, B$ is the modulation bandwidth.

In the $M Q A M-b a s e d$ connection, $P_{t}$ in (5) can be calculated according to the link budget relationship as follows,

$$
P_{t}=\frac{(4 \pi)^{2} M_{l} N_{r}}{G_{t} G_{r} \lambda^{2}} \cdot \frac{\bar{E}_{b}}{N_{0}} R_{b} d^{\kappa},
$$


where $\kappa$ is the channel path loss exponent, $d$ is the distance between transmitter and receiver, $G_{t}$ and $G_{r}$ are the transmitter and receiver antenna gains respectively, $M_{l}$ is the link margin which indicates the difference between the receiver sensitivity and the actual received power, $N_{r}$ is the single-sided power spectral density of the receiver noise, $\lambda$ is the carrier wavelength, and $\bar{E}_{b} / N_{0}$ is the normalized average energy per bit necessary for a given BER specification to the noise spectral density.

As referred to [23], the average $\bar{E}_{b} / N_{0}$ of the intra-cluster communication with a square constellation MQAM in AWGN channel is given by

$$
\left.\frac{\bar{E}_{b}}{N_{0}}\right|_{\text {intra }} \doteq \frac{M-1}{3 \log _{2} M}\left[Q^{-1}\left(\frac{\bar{P}_{B E R}^{\text {intra }} \log _{2} M}{4(1-1 / \sqrt{M})}\right)\right]^{2}
$$

where $Q(x)=\int_{x}^{\infty} \frac{1}{2 \pi} e^{-\frac{u^{2}}{2}} d u$ and $\bar{P}_{B E R}^{i n t r a}$ is the average BER of intra-cluster communication.

In [24], the average $\bar{E}_{b} / N_{0}$ of the inter-cluster communication with a square constellation MQAM in Rayleigh fading channel is given by

$$
\begin{aligned}
& \left.\frac{\bar{E}_{b}}{N_{0}}\right|_{\text {inter }} \doteq N_{T} \frac{2(M-1)}{3 \log _{2} M} \\
& \times\left[\frac { 1 } { 4 } \left(\frac{4(1-1 / \sqrt{M})\left(\begin{array}{c}
2 N_{T} N_{R}+1 \\
N_{T} N_{R}
\end{array}\right)}{\left.\left.\bar{P}_{B E R}^{\text {inter } \log _{2} M}\right)^{\frac{1}{N_{T} N_{R}}}-1\right]},\right.\right.
\end{aligned}
$$

where $N_{T}$ and $N_{R}$ are the number of transmission and reception devices and $\bar{P}_{B E R}^{i n t e r}$ is the average BER of inter-cluster communication.

\subsection{Power Consumption and Active Time in the Transmission}

\subsubsection{Data collection phase}

Data collection phase: The $\mathrm{CH}$, in this phase, in each cluster acts as the receiver dispersing the receiving path power consumption, while all CMs, SCoops and RCoops transmit data to their relating $\mathrm{CH}$, dissipating the transmitting path power consumption. According to the assumption of squared power path loss, the power consumption per bit for the data collection phase of devices in the $i$-th cluster can be expressed as follow,

$$
\begin{aligned}
& P_{C H}^{D C}(i)=P_{c r}, \\
& P_{C M}^{D C}(i, j)=\left.\frac{(4 \pi)^{2} M_{l} N_{r}}{G_{t} G_{r} \lambda^{2}} R_{b} d_{j, i}^{2} \cdot \frac{\bar{E}_{b}}{N_{0}}\right|_{\text {intra }}+P_{c t}, \\
& P_{S C o o p}^{D C}(i, m)=\left.\frac{(4 \pi)^{2} M_{l} N_{r}}{G_{t} G_{r} \lambda^{2}} R_{b} d_{m, i}^{2} \cdot \frac{\bar{E}_{b}}{N_{0}}\right|_{\text {intra }}+P_{c t}, \\
& P_{R \text { Coop }}^{D C}(i, k)=\left.\frac{(4 \pi)^{2} M_{l} N_{r}}{G_{t} G_{r} \lambda^{2}} R_{b} d_{k, i}^{2} \cdot \frac{\bar{E}_{b}}{N_{0}}\right|_{\text {intra }}+P_{c t},
\end{aligned}
$$


where $j \in\left[1, \cdots, \mathcal{N}_{C M}(i)\right], m \in\left[1, \cdots, \mathcal{N}_{\text {SCoop }}(i)\right]$ and $k \in\left[1, \cdots, \mathcal{N}_{R \text { Coop }}(i)\right]$. Similarly, the active time of devices in the $i$-th cluster for the data collection phase can be expressed as follows,

$$
\begin{aligned}
& T_{C H}^{D C}(i)=\left(\mathcal{N}_{C M}(i)+\mathcal{N}_{\text {SCoop }}(i)+\mathcal{N}_{R \text { Coop }}(i)\right) \frac{L}{R_{b}}, \\
& T_{C M}^{D C}(i, j)=\frac{L}{R_{b}} \\
& T_{S \text { Coop }}^{D C}(i, m)=\frac{L}{R_{b}} \\
& T_{R \text { Coop }}^{D C}(i, k)=\frac{L}{R_{b}},
\end{aligned}
$$

where $L$ is the amount of data in bits.

The $\mathrm{CH}$ performs data aggregation technique, after collecting data from all non- $\mathrm{CH}$ devices. In [25], the amount of data after aggregation is,

$$
L_{\text {agg }}(i)=\frac{\left(1+\mathcal{N}_{C M}(i)+\mathcal{N}_{\text {SCoop }}(i)+\mathcal{N}_{R \text { Coop }}(i)\right)}{\left(\mathcal{N}_{C M}(i)+\mathcal{N}_{\text {SCoop }}(i)+\mathcal{N}_{R \text { Coop }}(i)\right) \gamma+1} L,
$$

where $\gamma$ is the aggregation factor.

\subsubsection{Local broadcasting phase}

The CH in the $i$-th cluster, in the local broadcasting phase, acts as transmitter to broadcast the aggregated data to all SCoops, dissipating the transmitting path power consumption. Also all SCoops receive data information from the $\mathrm{CH}$, dissipating the receiving path power consumption. Due to the broadcast nature of the wireless channel, if the SCoop with the maximum distance from $\mathrm{CH}$ in the $i$-th cluster denoted by $d_{\max }(i)$ can receive the data from the $\mathrm{CH}$, the other SCoops can simultaneously receive these data. Then the power consumption per bit of devices for the local broadcasting phase in the $i$-th cluster can be expressed as follows,

$$
\begin{aligned}
& P_{C H}^{L B}(i)=\left.\frac{(4 \pi)^{2} M_{l} N_{r}}{G_{t} G_{r} \lambda^{2}} R_{b} d_{\text {max }}^{2}(i) \cdot \frac{\bar{E}_{b}}{N_{0}}\right|_{\text {intra }}+P_{c t}, \\
& P_{C M}^{L B}(i, j)=0, \\
& P_{S C o o p}^{L B}(i, m)=P_{c r}, \\
& P_{R \text { Coop }}^{L B}(i, k)=0,
\end{aligned}
$$

where $j \in\left[1, \cdots, \mathcal{N}_{C M}(i)\right], m \in\left[1, \cdots, \mathcal{N}_{\text {SCoop }}(i)\right]$ and $k \in\left[1, \cdots, \mathcal{N}_{R \text { Coop }}(i)\right]$. Similarly, the active time of devices in the $i$-th cluster for the local broadcasting 
phase can be stated as follows,

$$
\begin{aligned}
& T_{C H}^{L B}(i)=\frac{L_{a g g}(i)}{R_{b}}, \\
& T_{C M}^{L B}(i, j)=0, \\
& T_{S \text { Coop }}^{L B}(i, m)=\frac{L_{a g g}(i)}{R_{b}}, \\
& T_{R \text { Coop }}^{L B}(i, k)=0 .
\end{aligned}
$$

\subsubsection{Long-haul transmission phase}

In this phase, the $\mathrm{CH}$ and SCoops in the $i$-th cluster jointly transmit the MIMO modulated data to their adjoining $(i+1)$-th cluster or the capillary gateway, dissipating the transmitting path power consumption, while the $\mathrm{CH}$ and RCoops in the $(i+1)$-th cluster receive the MIMO modulated data, dissipating the receiving path power consumption. In wireless channels, the long-haul distance in the CMIMO communications represents the maximum distance between the transmission device in the $i$-th cluster and the reception devices in the $(i+1)$-th cluster. Denote these maximum distances as $d_{\max }^{\kappa_{\max }}(i)$ and $d_{\max }^{\kappa_{\max }}(m)$ for the $\mathrm{CH}$ and the SCoop respectively. Hence, at the transmission side for the

long-haul transmission phase, the power consumption per bit of devices in the $i$-th cluster can be expressed as follows,

$$
\begin{aligned}
& P_{C H}^{L H}(i)=\left.\frac{(4 \pi)^{2} M_{l} N_{r}}{G_{t} G_{r} \lambda^{2}} R_{b}^{e f f} d_{\max }^{\kappa_{\max }}(i) \cdot \frac{\bar{E}_{b}}{N_{0}}\right|_{\text {inter }}+P_{c t}, \\
& P_{C M}^{L H}(i, j)=0, \\
& P_{S \text { Coop }}^{L H}(i, m)=\left.\frac{(4 \pi)^{2} M_{l} N_{r}}{G_{t} G_{r} \lambda^{2}} R_{b}^{e f f} d_{\max }^{\kappa_{\max }}(m) \cdot \frac{\bar{E}_{b}}{N_{0}}\right|_{\text {inter }}+P_{c t}, \\
& P_{R \text { Coop }}^{L H}(i, k)=0,
\end{aligned}
$$

where $j \in\left[1, \cdots, \mathcal{N}_{C M}(i)\right], m \in\left[1, \cdots, \mathcal{N}_{S C \text { oop }}(i)\right]$ and $k \in\left[1, \cdots, \mathcal{N}_{R C o o p}(i)\right]$ and $R_{b}^{e f f}$ is the effective system bit rate as evaluated in (16).

In [26], authors introduced a training overhead into the CMIMO scheme for the purpose of channel estimation. The number of required training symbols is proportional to the number of transmit antennas. Hence, the packet size of long-haul transmission is given by,

$$
L_{c}(i)=\frac{F_{\text {block }}}{F_{\text {block }}-\rho_{\text {train }}\left(\mathcal{N}_{\text {SCoop }}+1\right)} \sum_{q=1}^{i} L_{\text {agg }}(q),
$$

where $F_{\text {block }}$ is the block size of STBC code, $\rho_{\text {train }}\left(\mathcal{N}_{\text {SCoop }}+1\right)$ is the number of training symbols in each block. According to [27], the effective system bit rate is given by,

$$
R_{b}^{\text {eff }}=\frac{F_{\text {block }}-\rho_{\text {train }}\left(\mathcal{N}_{\text {SCoop }}+1\right)}{F_{\text {block }}} R_{b} .
$$


Then, for the long-haul transmission phase, the active time of devices in the $i$-th cluster can be expressed as follows,

$$
\begin{aligned}
& T_{C H}^{L H}(i)=\frac{L_{c}(i)}{R_{b}^{e f f}}, \\
& T_{C M}^{L H}(i, j)=0, \\
& T_{S \text { Coop }}^{L H}(i, m)=\frac{L_{c}(i)}{R_{b}^{e f f}}, \\
& T_{R \text { Coop }}^{L H}(i, k)=0 .
\end{aligned}
$$

And at the reception side, the power consumption per bit of devices in the $(i+1)$-th cluster can be expressed as follows,

$$
\begin{aligned}
& P_{C H}^{L H}(i+1)=P_{c r}, \\
& P_{C M}^{L H}(i+1, j)=0, \\
& P_{S C o o p}^{L H}(i+1, m)=0, \\
& P_{R \text { Coop }}^{L H}(i+1, k)=P_{c r},
\end{aligned}
$$

where $j \in\left[1, \cdots, \mathcal{N}_{C M}(i+1)\right], m \in\left[1, \cdots, \mathcal{N}_{\text {SCoop }}(i+1)\right]$ and $k \in\left[1, \cdots, \mathcal{N}_{R \text { Coop }}(i+\right.$ $1)]$. Consistently, for the long-haul transmission phase, the active time of devices in the $(i+1)$-th cluster can be expressed as follows,

$$
\begin{aligned}
& T_{C H}^{L H}(i+1)=\left(\mathcal{N}_{R \text { Coop }}(i)+1\right) \frac{L_{c}(i)}{R_{b}^{e f f}}, \\
& T_{C M}^{L H}(i+1, j)=0, \\
& T_{S \text { Coop }}^{L H}(i+1, m)=0, \\
& T_{R \text { Coop }}^{L H}(i+1, k)=\left(\mathcal{N}_{R \text { Coop }}(i)+1\right) \frac{L_{c}(i)}{R_{b}^{e f f}} .
\end{aligned}
$$

\subsubsection{Local forwarding phase}

In this phase, $\mathrm{CH}$ in the $(i+1)$-th cluster receives long-haul MIMO data from all RCoops in the same cluster, dissipating the receiving path power consumption. In the meantime, RCoops transmit data to their $\mathrm{CH}$, dissipating the transmitting path power consumption. As a result, for the local forwarding phase, the power consumption per bit of devices in the $(i+1)$-th cluster can be expressed as follows,

$$
\begin{aligned}
& P_{C H}^{L F}(i+1)=P_{c r}, \\
& P_{C M}^{L F}(i+1, j)=0, \\
& P_{S \text { Coop }}^{L F}(i+1, m)=0 \\
& P_{R \text { Coop }}^{L F}(i+1, k)=\left.\frac{(4 \pi)^{2} M_{l} N_{r}}{G_{t} G_{r} \lambda^{2}} R_{b} d_{k, i+1}^{2} \cdot \frac{\bar{E}_{b}}{N_{0}}\right|_{\text {intra }}+P_{c t},
\end{aligned}
$$


where $j \in\left[1, \cdots, \mathcal{N}_{C M}(i+1)\right], m \in\left[1, \cdots, \mathcal{N}_{\text {SCoop }}(i+1)\right]$ and $k \in\left[1, \cdots, \mathcal{N}_{R \text { Coop }}(i+\right.$ 1)]. Similarly, for the local forwarding phase, the active time of devices in the $(i+1)$-th cluster can be expressed as follows,

$$
\begin{aligned}
& T_{C H}^{L F}(i+1)=\mathcal{N}_{R \text { Coop }}(i+1) \frac{L_{c}(i)}{R_{b}^{e f f}}, \\
& T_{C M}^{L F}(i+1, j)=0, \\
& T_{S C \text { oop }}^{L F}(i+1, m)=0, \\
& T_{R \text { Coop }}^{L F}(i+1, k)=\frac{L_{c}(i)}{R_{b}^{e f f}} .
\end{aligned}
$$

\subsection{Problem Formulation}

The average current required to power a device during period $\left[t_{s}, t_{e}\right]$ can be obtained by [28],

$$
\bar{I}_{c}=\frac{P_{t o t a l}}{\phi V},
$$

where $P_{\text {total }}$ is the overall power consumption of period $\left[t_{s}, t_{e}\right]$, while $\phi$ and $V$, respectively, denote the DC-DC converter output efficiency and voltage.

The overall power consumption $P_{\text {total }}$ of device $n$ is expressed as

$$
P_{\text {total }}(n)=P^{D C}(n)+P^{L B}(n)+P^{L F}(n)+P^{L H}(n)
$$

and $P^{D C}(n), P^{L B}(n), P^{L F}(n)$ and $P^{L H}(n)$ can be obtained from (9), (12), (20), and (14) and (18) respectively.

The overall active time $T_{\text {total }}$ of device $n$ during the transmission is expressed as

$$
T_{\text {total }}(n)=T^{D C}(n)+T^{L B}(n)+T^{L F}(n)+T^{L H}(n)
$$

where $T^{D C}(n), T^{L B}(n), T^{L F}(n)$ and $T^{L H}(n)$ can be obtained from (10), (13), (21), and (17) and (19) respectively.

The battery operating time of device $n$ can then be expressed as,

$$
\begin{aligned}
& \frac{C_{a v l}\left(I_{c}(n), T_{o p}(n), t_{s}(n), t_{e}(n), \beta^{2}\right)}{\bar{I}_{c}(n)} \\
& =\frac{C_{\text {init }}(n) \phi V}{P_{\text {total }}(n)}-\left(t_{e}(n)-t_{s}(n)\right) \\
& -2 \sum_{k=1}^{\infty} \frac{e^{-\beta^{2} k^{2}\left(T_{o p}(n)-t_{e}(n)\right)}-e^{-\beta^{2} k^{2}\left(T_{o p}(n)-t_{s}(n)\right)}}{\beta^{2} k^{2}} .
\end{aligned}
$$

In this work, we assume $t_{s}(n)=0$, and $t_{e}(n)=T^{D C}(n)+T^{L B}(n)+T^{L F}(n)+$ $T^{L H}(n)$.

The cooperative coalition in the $i$-th cluster can be expressed as follows,

$$
\begin{aligned}
& \operatorname{SCoop}(i)=\left\{\operatorname{SCoop}_{1}(i), \cdots, \operatorname{SCoop}_{\mathcal{N}_{\text {SCoop }(i)}}(i)\right\}, \\
& R \operatorname{Roop}(i)=\left\{\operatorname{RCoop}_{1}(i), \cdots, \operatorname{RCoop}_{\mathcal{N}_{R C o o p}(i)}(i)\right\}, \\
& \mathcal{C}(i)=R \operatorname{Coop}(i) \cup S \operatorname{Coop}(i) \text {. }
\end{aligned}
$$


The research problem in this work is to find the optimum cooperative coalitions of all clusters in the routing path, denoted by $\mathcal{C}=\left\{\mathcal{C}(1), \cdots, \mathcal{C}(i), \cdots, \mathcal{C}\left(\mathcal{N}_{C H}\right)\right\}$, in order to maximize the average battery operating time $\bar{T}_{o p}^{a v g}$ under given BER threshold $\bar{P}_{B E R}^{T H R}$. Hence the research problem formulation can be expressed as,

$$
\underset{\mathcal{C}}{\operatorname{maximize}} \bar{T}_{a v g}^{o p}=\frac{\sum_{n=1}^{\mathcal{N}_{\text {total }}} T_{o p}(n)}{\mathcal{N}_{\text {total }}}
$$

\section{Description and Analysis of QPSO Algorithm}

In PSO, which is an evolutionary computing technique based on the bird flocking principle, a swarm consists of several particles and each particle represents a candidate solution to the optimization problem. To find the particle position, each particle flies in the search space and updates its best individual optimum position and global optimum position of the swarm by moving towards a better solution space. QPSO introduces quantum coding mechanism to encode each particle. It was tested on some benchmark functions and experimental results showed that QPSO outperforms PSO [29].

\subsection{Quantum Particle Swarm Optimization (QPSO)}

QPSO encodes each particle by a quantum bit (qubit). A qubit is defined in [30] as a pair of composite numbers $(\alpha, \beta)$, where $|\alpha|^{2}+|\beta|^{2}=1$ and $\alpha>0$, $\beta>0 .|\alpha|^{2}$ gives the probability that the quantum bit is found in ' $0^{\prime}$ state and $|\beta|^{2}$ gives the probability that the quantum bit is found in ' 1 ' state. Then the quantum velocity of particle $m$ at generation $t$ is defined as

$$
\mathbf{v}_{m}^{t}=\left[\begin{array}{cccc}
\alpha_{m 1}^{t} & \alpha_{m 2}^{t} & \cdots & \alpha_{m \mathcal{R}}^{t} \\
\beta_{m 1}^{t} & \beta_{m 2}^{t} & \cdots & \beta_{m \mathcal{R}}^{t}
\end{array}\right],
$$

where $\mathcal{R}$ is the dimension of the research problem and $m \in[1,2, \cdots, h], h$ is the number of particles.

Since $\beta_{m n}=\sqrt{1-\alpha_{m n}^{2}}$, we can simplify (28) as

$$
\mathbf{v}_{m}^{t}=\left[\begin{array}{llll}
\alpha_{m 1}^{t} & \alpha_{m 2}^{t} & \cdots & \alpha_{m R}^{t}
\end{array}\right] .
$$

The quantum particle position according to (29) can be expressed as

$$
x_{m n}^{t}=\left\{\begin{array}{lll}
1 & \text { if } & \delta_{m n}>\left(v_{m n}^{t}\right)^{2} \\
0 & \text { if } & \delta_{m n} \leq\left(v_{m n}^{t}\right)^{2}
\end{array},\right.
$$

where $\delta_{m n} \in[0,1]$ is a uniform random number between 0 and 1 . In this paper, as shown in Fig. 5, the quantum position of a particle is composed of all possible cooperative coalitions candidates in the routing path. $S C_{o o p}$ cand $(n)$

represents SCoops candidates in Cluster $n$, which is the set of all non-CH devices in Cluster $n$. Furthermore, $R C \operatorname{oop}_{\text {cand }}(n)$ share the same candidates set with 
$S_{C o o p}$ cand $(n)$, which means that every non-CH device in Cluster $n$ has the potential to be a SCoop and RCoop. The value of quantum position indicates whether the device $n$ in particle $m$ is a member of the cooperative coalitions: $x_{m n}^{t}=1$ represents that the candidate $n$ in particle $m$ is a SCoop/RCoop at generation $t$; otherwise, it is a CM at generation $t$. Therefore each particle in this paper represents a possible solution of particular cooperative coalitions, and the fitness value of each particle can be obtained by (27).

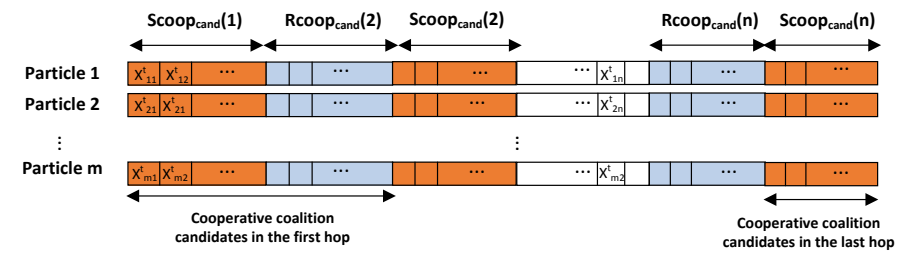

Figure 5: Particle position representation

Denote the fitness value of particle $m$ at generation $t$ to be $f_{m}^{t}$, then the local individual optimum fitness value $f_{m}^{p b e s t}$ which is defined as the maximum fitness value of particle $m$ from the first generation to the current generation $t$. Accordingly, the corresponding local individual optimum position $\mathbf{p}_{m}$ is defined as follows,

$$
\begin{aligned}
& f_{m}^{\text {pbest }}= \max \left\{f_{m}^{1}, f_{m}^{2}, \cdots, f_{m}^{t}\right\}, \\
& \mathbf{p}_{m}=\mathbf{x}_{m}^{\text {pbest }} .
\end{aligned}
$$

Likewise, the global optimum fitness value $f_{\text {gbest }}$ that is defined as the maximum local individual optimum fitness value of all particles and the corresponding global optimum position $\mathbf{p}_{g}$ is defined as below,

$$
\begin{gathered}
f_{\text {gbest }}=\max \left\{f_{1}^{\text {pbest }}, \cdots, f_{m}^{\text {pbest }}, \cdots, f_{h}^{\text {pbest }}\right\}, \\
\mathbf{p}_{g}=\mathbf{p}_{\text {gbest }} .
\end{gathered}
$$

The quantum rotation angle $\theta_{m n}^{t+1}$, at generation $t+1$, is updated by

$$
\theta_{m n}^{t+1}=k_{1}\left(p_{m n}-x_{m n}^{t}\right)+k_{2}\left(p_{g n}-x_{m n}^{t}\right),
$$

where $k_{1}$ and $k_{2}$ are positive learning factors of cognitive and social acceleration, respectively. The cognitive acceleration factor represents the attraction that a particle has toward its own success while the social acceleration factor represents the attraction that a particle has toward the success of its neighbours. Referring to [31], we set $k_{1}=\frac{1}{5} \zeta_{1}$ and $k_{2}=\frac{4}{5} \zeta_{2}$ where $\zeta_{1}$ and $\zeta_{2}$ are Gaussian distributed random numbers with zero mean and unit variance.

The updated velocity of the quantum particle $m$ at $t+1$ generation is

$$
v_{m n}^{t+1}=\left\{\begin{array}{c}
\sqrt{1-\left(v_{m n}^{t}\right)^{2}}, \quad \text { if } \theta_{m n}^{t+1}=0 \text { and } \delta=c_{1} \\
\left|v_{m n}^{t} \cos \theta_{m n}^{t+1}-\sqrt{1-\left(v_{m n}^{t}\right)^{2}} \sin \theta_{m n}^{t+1}\right|, \text { otherwise }
\end{array}\right.
$$


where $\delta$ is a uniform random number between 0 and 1 , and $c_{1}$ is a constant which refers to the mutation probability, $c_{1} \in[0,1 / \mathcal{R}]$.

\subsection{QPSO Cooperative Coalitions Selection}

The multi-hop-based transmission procedure using CMIMO scheme in clusterbased capillary networks is summarized in Algorithm 1 and the cooperative coalitions selection process based on QPSO is executed by the capillary gateway and summarized in Algorithm 2. Denote the number of routing paths in the capillary network to be $\mathcal{N}_{\text {route }}$, the number of candidates set to be $\mathcal{N}_{\text {Cand }}$, and the number of generations to be $T_{\max }$. below:

$\left[R_{1,1}\right]$ The different steps involved in Algorithm 2 for each route are given

- Step 1: Initialize the positions of all particles (potential solutions) in the population randomly (lines 2 to 5 ), and evaluate their fitness values and positions (lines 6 to 8 ).

- Step 2: Assign the current fitness value to gbest and assign the current coordinates to gbest coordinates (lines 9 to 10 ).

- Step 3: Update the rotation, velocity, and position of all particles (lines 12 to 16$)$.

- Step 4: Evaluate the fitness value of all particles (line 17).

- Step 5: Compare the personal best (pbest) of every particle with its current fitness value. If the current fitness value is better, then assign the current fitness value to pbest and assign the current coordinates to pbest coordinates (lines 18 to 20 ).

- Step 6: Determine the current best fitness value in the whole population and its coordinates. If the current best fitness value is better than global best (gbest), then assign the current best fitness value to gbest and assign the current coordinates to gbest coordinates (lines 21 to 24). Note that this is the step that allows the algorithm to maximize the network lifetime by choosing the particle (i.e. potential solution) that has the maximum $\bar{T}_{a v g}^{o p}$ as per (27) and (33).

- Step 7: Repeat steps 3-6 until the number of generations reaches $T_{\max }$.

$\left[R_{2,2}\right]\left[R_{1,2}\right]$ Although Algorithm 2 is executed by the capillary gateway, that can be considered as having extensive computational power, it is interesting to assess the complexity of the algorithm. In this complexity analysis, $h$ and $T_{\max }$ are considered to be constants. The for loop of line 1 is executed $\mathcal{N}_{\text {route }}$ times. The complexity of the lines 2 to 8 is $O\left(\mathcal{N}_{\text {Cand }}+\mathcal{N}_{\text {total }}\right)$. The complexity of lines 9 and 10 is $O(1)$. Finally, the complexity of the lines from 11 to 24 is $O\left(\mathcal{N}_{\text {Cand }}+\mathcal{N}_{\text {total }}\right)$. Hence the overall complexity is $O\left(\mathcal{N}_{\text {route }}\left[\mathcal{N}_{\text {Cand }}+\mathcal{N}_{\text {total }}\right]\right)$. Since $\mathcal{N}_{\text {Cand }}<\mathcal{N}_{\text {total }}$ and $\mathcal{N}_{\text {route }} \leq \mathcal{N}_{\text {total }}$, the overall complexity of Algorithm 2 
is $O\left(\mathcal{N}_{\text {total }}^{2}\right)$. Note that this is a worst case scenario complexity as in practice the number of routes is at maximum equal to the number of $\mathrm{CH}$ s and in practice the number of $\mathrm{CHs}$ is much smaller than $\mathcal{N}_{\text {total }}$.

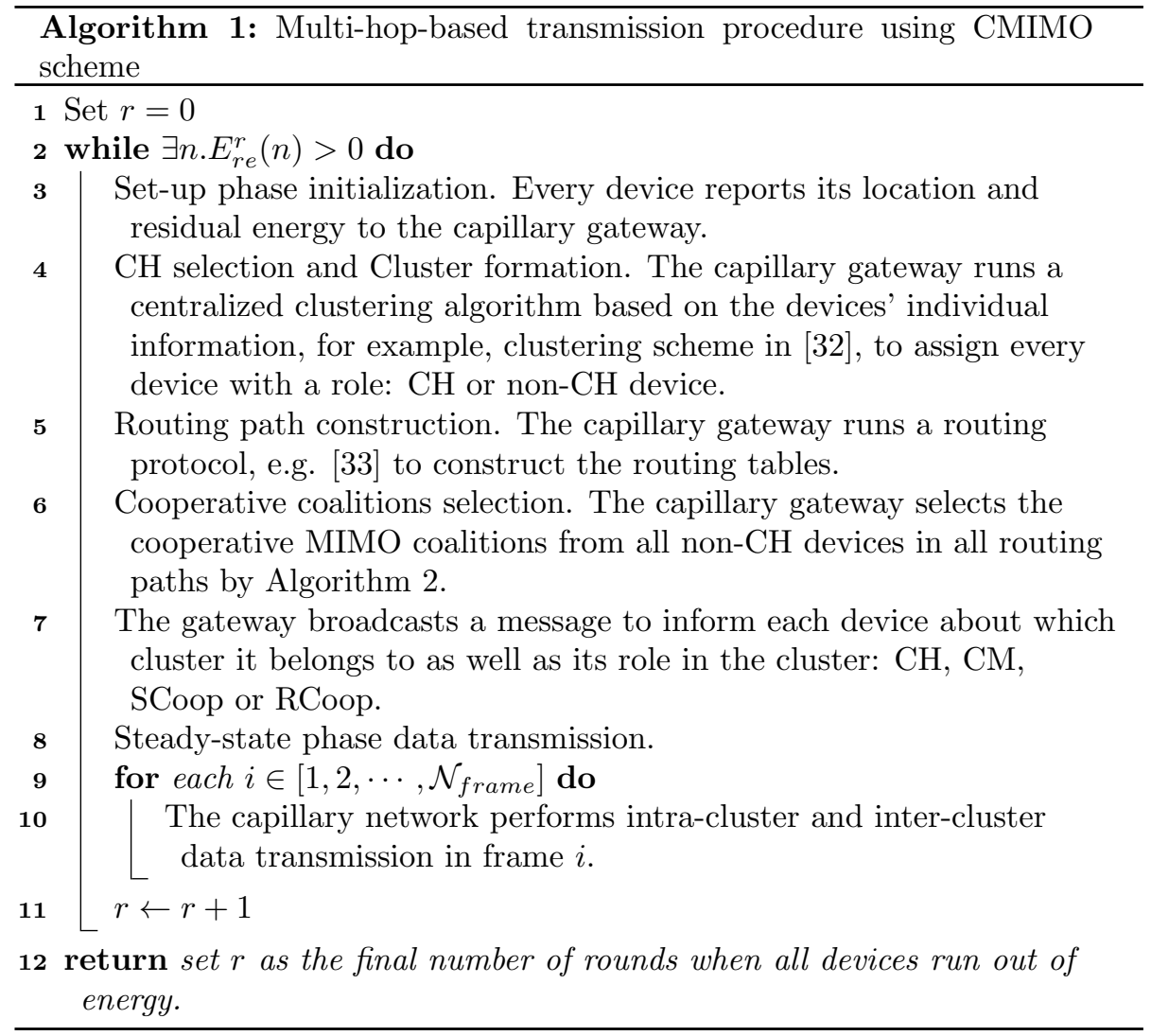

\section{Simulation}

In this paper, for simplicity, we assume a scenario with three clusters in one routing path, i.e. 3 hops routing. Each cluster consists of 10 devices which are randomly distributed within a circle of $25 \mathrm{~m}$ radius. Furthermore, all devices are powered by one AAA Li-FeS2 battery, which has 1.5-volt nominal voltage and $1200 \mathrm{mAh}$ nominal capacity.

$\left[R_{1,3}\right]$ Similar works that try to address the same problem are [32] and [34]. However, [32] does not consider the cooperation mechanism, while [34] is a CMISO system where the focus is on the cooperative transmission process only. Hence, we simulate the $2 \times 2 \mathrm{MIMO}$ and $3 \times 3 \mathrm{MIMO}$ systems as reference so that we can compare our work with the peer work. We simulate the transmission in one round. The system parameters are given in Table 1 . 


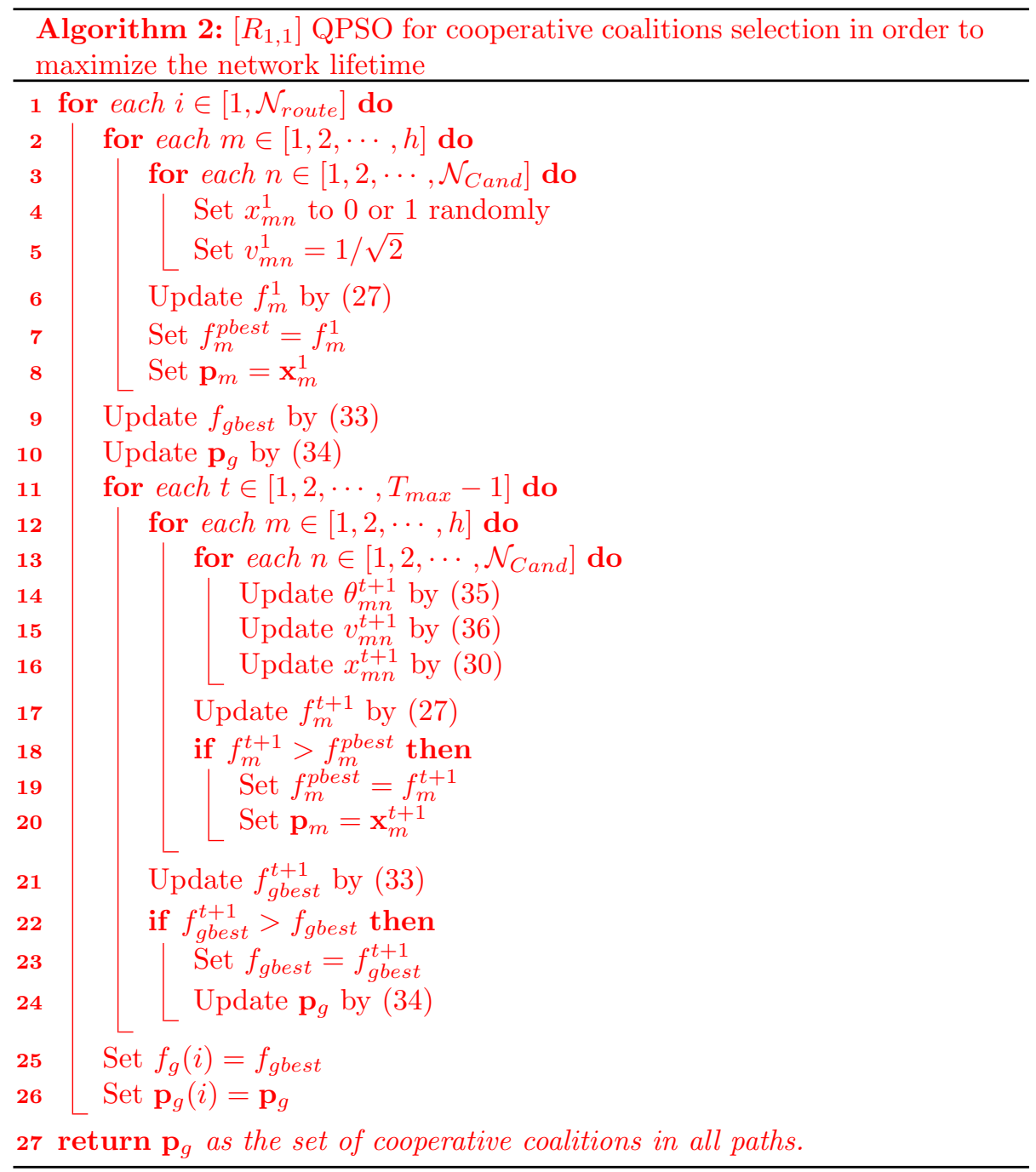

Table 1: System Parameters

\begin{tabular}{|l|l|l|}
\hline$M_{l}=40 d B$ & $N_{f}=10 d B$ & $N_{r}=-161 d B m / H z$ \\
\hline$B=10 k H z$ & $G_{T} G_{R}=5 d B i$ & $\lambda=0.12 m$ \\
\hline $\mathcal{N}_{\text {frame }}=25$ & $\eta=0.47$ & $L=1000 b i t$ \\
\hline $\mathcal{N}_{\text {total }}=25$ & $\beta=0.5$ & $P_{L N A}=20 \mathrm{~mW}$ \\
\hline$P_{D A C}=15.5 \mathrm{~mW}$ & $P_{\text {mix }}=30.3 \mathrm{~mW}$ & $P_{\text {filt }}=2.5 \mathrm{~mW}$ \\
\hline$P_{\text {filr }}=2.5 \mathrm{~mW}$ & $P_{\text {IFA }}=3 \mathrm{~mW}$ & $P_{A D C}=9.8 \mathrm{~mW}$ \\
\hline$F_{\text {block }}=200$ & $\rho_{\text {train }}=2$ & $M=16$ \\
\hline
\end{tabular}


First, one of the main difficulties of applying an evolutionary algorithm to a given problem is to decide on an appropriate set of parameter values, such as optimum number of particles and optimum number of generations [35]. Denote the optimum number of generations to be $T_{\text {opt }}$, which shows the number of generations required to first produce the final optimum global fitness value. In addition, as referred to [36], the function evaluations denoted by $\mathbb{F E}$ is defined as $\mathbb{F E}=T_{\text {opt }} \times h$, which indicates the algorithm complexity with respect to different number of particles. Table 2 shows the optimum number of generations and function evaluations for different number of particles to converge to the same optimum global fitness value. The simulation iteration is set to be 100 times. In order to emphasize the convergence trend in Table 2, Fig. 6 shows the convergence time with respect to both optimum number of generations and function evaluations.

Table 2: Algorithm complexity analysis of QPSO

\begin{tabular}{|l|l|l|l|l|l|}
\hline Number of particle $(h)$ & 5 & 10 & 15 & 20 & 25 \\
\hline Optimum number of generations $\left(T_{\text {opt }}\right)$ & 954.3 & 256.6 & 241.1 & 243.1 & 247.8 \\
\hline Function Evaluations $(\mathbb{F E})$ & 4771.5 & 2566 & 3616.5 & 4863.3 & 9165.8 \\
\hline \hline Number of particle $(h)$ & 30 & 35 & 40 & 45 & 50 \\
\hline Optimum number of generations $\left(T_{\text {opt }}\right)$ & 232.2 & 205.9 & 228.9 & 206.9 & 224.7 \\
\hline Function Evaluations $(\mathbb{F E})$ & 6966 & 7208.8 & 9157.3 & 9313.5 & 11236.7 \\
\hline
\end{tabular}

In Fig. 6, the optimum number of generations decreases dramatically when the number of particles increases from 5 to 10. This is because more particles means better opportunity to find the optimum fitness value. However, as the number of particles increases from 10 to 50, the optimum number of generations varies within a small range between 205.9 to 256.6 , therefore, the performance of QPSO is not sensitive to higher number of particles. Focusing on the function evaluations, it can be seen that the minimum $\mathbb{F E}$ is achieved at the point where the number of particle is 10 and the optimum number of generation is 256.6. Therefore, in order to reduce the algorithm complexity, we set the number of particles to be 10 and number of generations to be 300 in the following simulation.

Next, the long-haul distance has been taken into consideration as shown in Fig. 7. We set $\bar{P}_{B E R}^{T H R}$ to be $10^{-4}$. It is observed that the proposed QPSO cooperative coalitions selection algorithm outperforms the $2 \times 2 \mathrm{MIMO}$ and $3 \times 3$ MIMO in terms of average battery operating time by approximately $20 \%$. This is because the proposed algorithm can select the optimum cooperative coalition in every link dynamically. In addition, the $2 \times 2$ MIMO system outperforms $3 \times 3$ MIMO system in a short long-haul distance around $100 \mathrm{~m}$, due to more circuit energy consumption of $3 \times 3$ MIMO system. Note that with the increase of long-haul distance, the average battery capacity decreases. This is because more RCoops and SCoops are selected to support the long-haul transmission.

Table 3 shows the size of cooperative coalition in each link in terms of longhaul distance. We can see that the size of cooperative coalitions increases from link 1 to link 3 on account of the same long-haul distance, due to the increment 


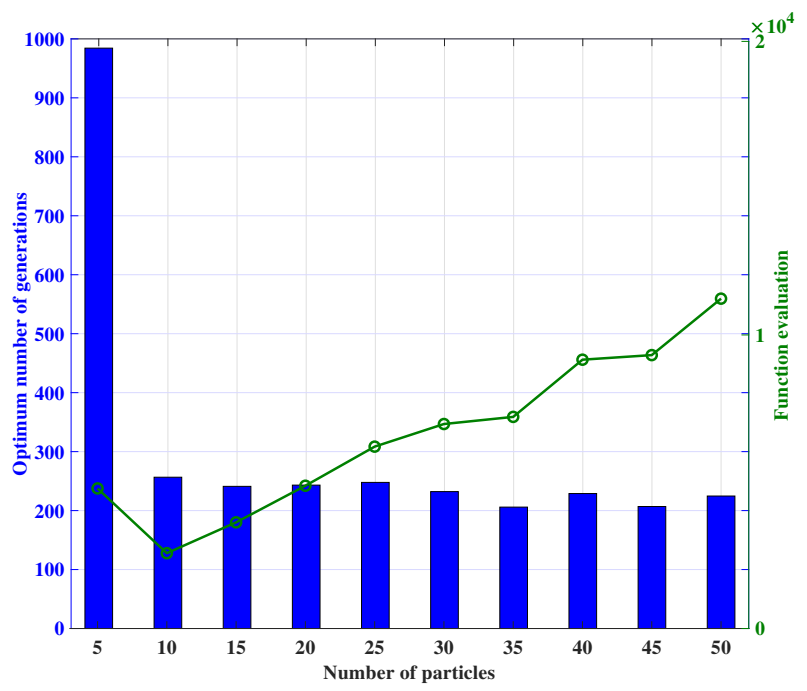

Figure 6: Time complexity analysis of QPSO

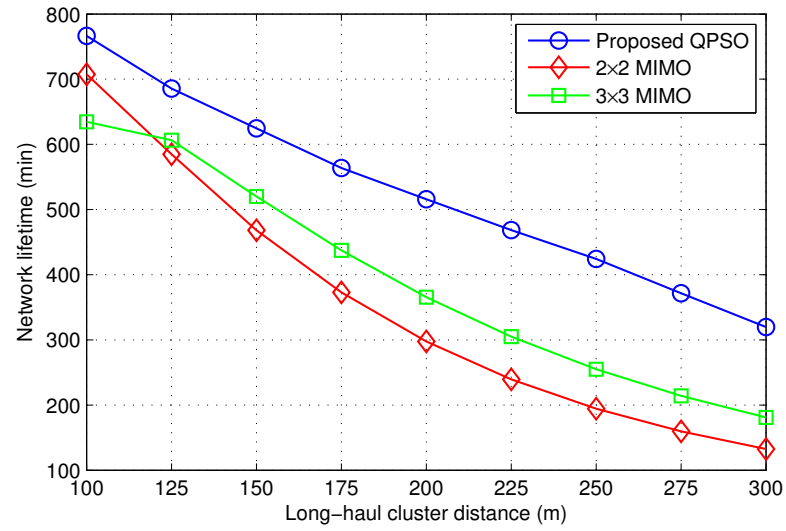

Figure 7: Network lifetime vs. long-haul distance between CHs

of the packet size in the long-haul transmission. Furthermore, it is clear that, the size of the cooperative coalition also increases in terms of long-haul distance. However, the size of the cooperative coalition remains the same when the longhaul distance becomes larger. This is because no optimum SCoops and RCoops can be selected.

Fig. 8 illustrates the average battery capacity in terms of BER threshold $\bar{P}_{B E R}^{T H R}$. The long-haul distance is set to be $100 \mathrm{~m}$. Aforementioned, the proposed QPSO algorithm can select cooperative coalitions in each link dynamically, and 
Table 3: Size of cooperative coalitions in multi-hop routing

\begin{tabular}{|c|c|c|c|}
\hline Long-haul distance $(\mathrm{m})$ & Link 1 & Link 2 & Link 3 \\
\hline 100 & $1 \times 2 \mathrm{MIMO}$ & $2 \times 2 \mathrm{MIMO}$ & $3 \times 1 \mathrm{MIMO}$ \\
\hline 125 & $1 \times 2 \mathrm{MIMO}$ & $2 \times 3 \mathrm{MIMO}$ & $3 \times 1 \mathrm{MIMO}$ \\
\hline 150 & $1 \times 2 \mathrm{MIMO}$ & $2 \times 3 \mathrm{MIMO}$ & $4 \times 1 \mathrm{MIMO}$ \\
\hline 175 & $1 \times 2 \mathrm{MIMO}$ & $3 \times 3 \mathrm{MIMO}$ & $4 \times 1 \mathrm{MIMO}$ \\
\hline 200 & $1 \times 2 \mathrm{MIMO}$ & $3 \times 3 \mathrm{MIMO}$ & $4 \times 1 \mathrm{MIMO}$ \\
\hline 225 & $1 \times 2 \mathrm{MIMO}$ & $3 \times 3 \mathrm{MIMO}$ & $5 \times 1 \mathrm{MIMO}$ \\
\hline 250 & $2 \times 2 \mathrm{MIMO}$ & $3 \times 4 \mathrm{MIMO}$ & $5 \times 1 \mathrm{MIMO}$ \\
\hline 275 & $2 \times 3 \mathrm{MIMO}$ & $3 \times 4 \mathrm{MIMO}$ & $5 \times 1 \mathrm{MIMO}$ \\
\hline 300 & $2 \times 3 \mathrm{MIMO}$ & $3 \times 4 \mathrm{MIMO}$ & $5 \times 1 \mathrm{MIMO}$ \\
\hline
\end{tabular}

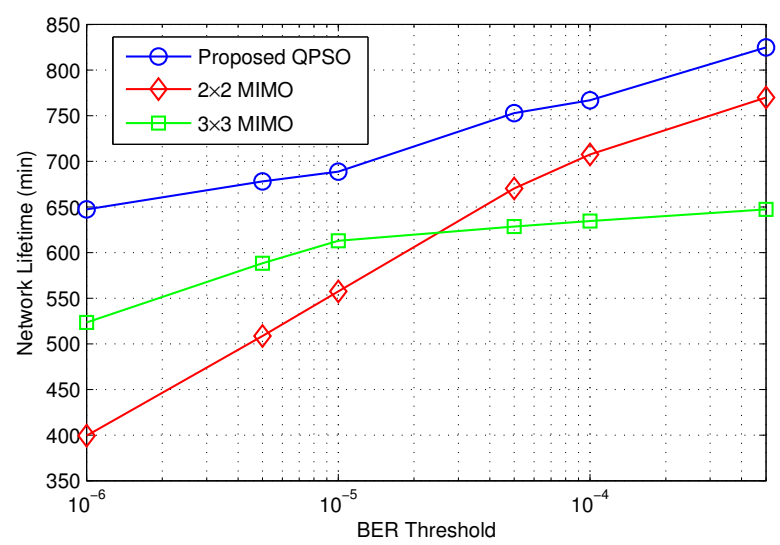

Figure 8: Network lifetime vs. BER threshold

hence it outperforms $2 \times 2 \mathrm{MIMO}$ and $3 \times 3 \mathrm{MIMO}$ systems. Furthermore, the $2 \times 2$ MIMO system can save more energy than $3 \times 3$ MIMO system under the same BER threshold after the BER threshold reaches $2.5 \times 10^{-5}$, because the size of optimum cooperative coalitions becomes smaller. We also investigate the size of the cooperative coalition in each link in terms of BER threshold in Table 4. Table 4 indicates that the proposed QPSO algorithm can select the cooperative coalitions dynamically to meet different BER requirements.

\section{Conclusion}

In this paper, we investigate the cooperative coalitions selection using QPSO algorithms with the aim of maximizing the average battery operating time in multi-hop and cluster-based IoT systems. We conclude that the proposed QPSO based cooperative coalitions selection algorithm can select the optimum cooperative devices dynamically to participate in the long-haul MIMO transmission. In particular, the proposed QPSO-based MIMO scheme outperforms $2 \times 2$ MIMO 
Table 4: Size of cooperative coalitions in multi-hop routing

\begin{tabular}{|c|c|c|c|}
\hline BER Threshold & Link 1 & Link 2 & Link 3 \\
\hline $10^{-6}$ & $1 \times 3 \mathrm{MIMO}$ & $3 \times 3 \mathrm{MIMO}$ & $5 \times 2 \mathrm{MIMO}$ \\
\hline $5 \times 10^{-6}$ & $1 \times 3 \mathrm{MIMO}$ & $3 \times 3 \mathrm{MIMO}$ & $5 \times 2 \mathrm{MIMO}$ \\
\hline $10^{-5}$ & $1 \times 3 \mathrm{MIMO}$ & $3 \times 2 \mathrm{MIMO}$ & $5 \times 2 \mathrm{MIMO}$ \\
\hline $5 \times 10^{-5}$ & $1 \times 2 \mathrm{MIMO}$ & $3 \times 2 \mathrm{MIMO}$ & $4 \times 2 \mathrm{MIMO}$ \\
\hline $10^{-4}$ & $1 \times 2 \mathrm{MIMO}$ & $2 \times 2 \mathrm{MIMO}$ & $3 \times 1 \mathrm{MIMO}$ \\
\hline $5 \times 10^{-4}$ & $1 \times 2 \mathrm{MIMO}$ & $3 \times 2 \mathrm{MIMO}$ & $3 \times 2 \mathrm{MIMO}$ \\
\hline
\end{tabular}

and $3 \times 3$ MIMO regarding average battery operating time irrespective of the target BER.

\section{References}

[1] S. Lucero, IoT platforms: enabling the internet of things, Tech. rep., IHS TECHNOLOGY (3 2016).

URL https://cdn.ihs.com/www/pdf/enabling-IOT.pdf

[2] M. T. Mardini, Y. Iraqi, N. Agoulmine, A survey of healthcare monitoring systems for chronically ill patients and elderly, Journal of Medical Systems 43 (3) (2019) 50. doi:10.1007/s10916-019-1165-0.

URL https://doi.org/10.1007/s10916-019-1165-0

[3] Y. Li, K. K. Chai, Y. Chen, J. Loo, Duty cycle control with joint optimisation of delay and energy efficiency for capillary machine-to-machine networks in 5G communication system, Transactions on Emerging Telecommunications Technologies 26 (1) (2015) 56-69. doi:10.1002/ett.2891.

[4] V. B. Mišić, J. Mišić, D. Nerandzic, Extending LTE to support machinetype communications, in: 2012 IEEE International Conference on Communications (ICC), 2012, pp. 6977-6981. doi:10.1109/ICC.2012.6364741.

[5] $\left[R_{1,5}\right]$ Y. Li, N. Yu, W. Zhang, W. Zhao, X. You, M. Daneshmand, Enhancing the performance of LEACH protocol in wireless sensor networks, in: 2011 IEEE Conference on Computer Communications Workshops (INFOCOM WKSHPS), 2011, pp. 223-228. doi:10.1109/INFCOMW.2011.5928813.

[6] $\left[R_{1,5}\right]$ Y. Li, C. Liao, Y. Wang, C. Wang, Energy-efficient optimal relay selection in cooperative cellular networks based on double auction, IEEE Transactions on Wireless Communications 14 (8) (2015) 4093-4104. doi:10.1109/TWC.2015.2416715.

[7] $\left[R_{1,5}\right]$ Y. Li, Z. Zhang, C. Wang, W. Zhao, H. Chen, Blind cooperative communications for multihop ad hoc wireless networks, IEEE Transactions on Vehicular Technology 62 (7) (2013) 3110-3122. doi:10.1109/TVT.2013.2256475. 
[8] $\left[R_{2,3}\right]$ T. Lv, Z. Lin, P. Huang, J. Zeng, Optimization of the energy-efficient relay-based massive IoT network, IEEE Internet of Things Journal 5 (4) (2018) 3043-3058. doi:10.1109/JIOT.2018.2829827.

[9] $\left[R_{2,3}\right]$ C. H. Liu, J. Fan, J. W. Branch, K. K. Leung, Toward QoI and energy-efficiency in internet-of-things sensory environments, IEEE Transactions on Emerging Topics in Computing 2 (4) (2014) 473-487. doi:10.1109/TETC.2014.2364915.

[10] $\left[R_{2,3}\right]$ J. Tang, D. K. C. So, N. Zhao, A. Shojaeifard, K. Wong, Energy efficiency optimization with SWIPT in MIMO broadcast channels for internet of things, IEEE Internet of Things Journal 5 (4) (2018) 2605-2619. doi:10.1109/JIOT.2017.2785861.

$[11]\left[R_{2,3}\right]$ C. H. Liu, B. Yang, T. Liu, Efficient naming, addressing and profile services in internet-of-things sensory environments, Ad Hoc Networks 18 (2014) 85 - 101. doi:https://doi.org/10.1016/j.adhoc.2013.02.008.

URL http://www.sciencedirect.com/science/article/pii/ S1570870513000280

[12] A. d. Coso, S. Savazzi, U. Spagnolini, C. Ibars, Virtual MIMO channels in cooperative multi-hop wireless sensor networks, in: 40th Annual Conference on Information Sciences and Systems, 2006, pp. 75-80. doi:10.1109/CISS.2006.286439.

[13] S. Park, W. Lee, D. Cho, Fair clustering for energy efficiency in a cooperative wireless sensor network, in: IEEE 75th Vehicular Technology Conference (VTC Spring), 2012, pp. 1-5. doi:10.1109/VETECS.2012.6240000.

[14] I. Ahmed, M. Peng, W. Wang, Uniform energy consumption through adaptive optimal selection of cooperative MIMO schemes in wireless sensor networks, in: VTC Spring 2008 - IEEE Vehicular Technology Conference, 2008, pp. 198-202. doi:10.1109/VETECS.2008.53.

[15] T. Scully, M. G. Madden, G. Lyons, Coalition calculation in a dynamic agent environment, in: Proceedings of the Twenty-first International Conference on Machine Learning, ICML '04, ACM, New York, NY, USA, 2004, pp. 93-. doi:10.1145/1015330.1015380.

URL http://doi.acm.org/10.1145/1015330.1015380

[16] J. Cao, T. Zhang, Z. Zeng, Y. Chen, K. K. Chai, Multi-relay selection schemes based on evolutionary algorithm in cooperative relay networks, International Journal of Communication Systems 27 (4) (2014) 571-591. arXiv:https://onlinelibrary.wiley.com/doi/pdf/10.1002/dac.2710, doi:10.1002/dac.2710.

URL https://onlinelibrary.wiley.com/doi/abs/10.1002/dac.2710

[17] J. Zhang, S. Ci, H. Sharif, M. Alahmad, Modeling discharge behavior of multicell battery, IEEE Transactions on Energy Conversion 25 (4) (2010) 1133-1141. doi:10.1109/TEC.2010.2048904. 
[18] J. N. Laneman, G. W. Wornell, Distributed space-time coded protocols for exploiting cooperative diversity in wireless networks, in: Global Telecommunications Conference, 2002. GLOBECOM '02. IEEE, Vol. 1, 2002, pp. 77-81 vol.1. doi:10.1109/GLOCOM.2002.1188045.

[19] W. R. Heinzelman, A. Chandrakasan, H. Balakrishnan, Energy-efficient communication protocol for wireless microsensor networks, in: Proceedings of the 33rd Annual Hawaii International Conference on System Sciences, 2000, pp. 10 pp. vol.2-. doi:10.1109/HICSS.2000.926982.

[20] D. N. Rakhmatov, S. B. K. Vrudhula, An analytical high-level battery model for use in energy management of portable electronic systems, in: IEEE/ACM International Conference on Computer Aided Design. ICCAD 2001. IEEE/ACM Digest of Technical Papers (Cat. No.01CH37281), 2001, pp. 488-493. doi:10.1109/ICCAD.2001.968687.

[21] S. Cui, A. J. Goldsmith, A. Bahai, Energy-efficiency of MIMO and cooperative MIMO techniques in sensor networks, IEEE Journal on Selected Areas in Communications 22 (6) (2004) 1089-1098. doi:10.1109/JSAC.2004.830916.

[22] T. H. Lee, The design of CMOS radio-frequency integrated circuits, 2nd edition, Communications Engineer 2 (4) (2004) 47-47.

[23] H. Dai, L. Xiao, Q. Zhou, Energy efficiency of MIMO transmission strategies in wireless sensor networks, in: International Conference on Computing, Communications and Control Technologies (CCCT), 2004.

[24] Y. Zhang, H. Dai, Energy-efficiency and transmission strategy selection in cooperative wireless sensor networks, Journal of Communications and Networks 9 (4) (2007) 473-481. doi:10.1109/JCN.2007.6182883.

[25] Y. Yuan, M. Chen, T. Kwon, A novel cluster-based cooperative MIMO scheme for multi-hop wireless sensor networks, EURASIP Journal on Wireless Communications and Networking 2006 (1) (2006) 072493. doi:10.1155/WCN/2006/72493.

URL https://doi.org/10.1155/WCN/2006/72493

[26] S. K. Jayaweera, Energy Analysis of MIMO Techniques in Wireless Sensor Networks, in: International conference on information sciences and systems, 2004.

[27] B. Hassibi, B. M. Hochwald, How much training is needed in multipleantenna wireless links?, IEEE Transactions on Information Theory 49 (4) (2003) 951-963. doi:10.1109/TIT.2003.809594.

[28] J. Zhang, S. Ci, H. Sharif, M. Alahmad, A battery-aware deployment scheme for cooperative wireless sensor networks, in: GLOBECOM 2009 - 2009 IEEE Global Telecommunications Conference, 2009, pp. 1-5. doi:10.1109/GLOCOM.2009.5425401. 
[29] S. M. Mikki, A. A. Kishk, Quantum particle swarm optimization for electromagnetics, IEEE Transactions on Antennas and Propagation 54 (10) (2006) 2764-2775. doi:10.1109/TAP.2006.882165.

[30] J. C. Hongyuan Gao, M. Diao, A simple quantum-inspired particle swarm optimization and its application, Information Technology Journal 10 (12) (2011) 2315-2321.

[31] J. Cao, T. Zhang, Z. Zeng, D. Liu, Interference-aware multiuser relay selection scheme in cooperative relay networks, in: 2013 IEEE Globecom Workshops (GC Wkshps), 2013, pp. 368-373. doi:10.1109/GLOCOMW.2013.6825015.

[32] L. Song, K. K. Chai, Y. Chen, J. Loo, S. Jimaa, J. Schormans, QPSObased energy-aware clustering scheme in the capillary networks for internet of things systems, in: 2016 IEEE Wireless Communications and Networking Conference, 2016, pp. 1-6. doi:10.1109/WCNC.2016.7564864.

[33] J. Yu, Y. Qi, G. Wang, X. Gu, A cluster-based routing protocol for wireless sensor networks with nonuniform node distribution, AEU - International Journal of Electronics and Communications 66 (1) (2012) $54-61$. doi:https://doi.org/10.1016/j.aeue.2011.05.002.

[34] B. Li, H. Li, W. Wang, Q. Yin, H. Liu, Performance Analysis and Optimization for Energy-Efficient Cooperative Transmission in Random Wireless Sensor Network, IEEE Transactions on Wireless Communications 12 (9) (2013) 4647-4657. doi:10.1109/TWC.2013.072313.121949.

[35] F. G. Lobo, C. F. Lima, Z. Michalewicz, Parameter Setting in Evolutionary Algorithms, 1st Edition, Springer Publishing Company, Incorporated, 2007.

[36] S. Chen, J. Montgomery, A. Bolufé-Röhler, Measuring the curse of dimensionality and its effects on particle swarm optimization and differential evolution, Applied Intelligence 42 (3) (2015) 514-526. doi:10.1007/s10489014-0613-2.

URL https://doi.org/10.1007/s10489-014-0613-2 
Liumeng Song (S'14) received the B.E. degree from Beijing University of Posts and Telecommunications, Beijing, China, in 2012, and the Ph.D. degree in wireless communications from Queen Mary University of London, London, U.K., in 2017. Her research interests include machine-to-machine (M2M), energy efficiency, clustering, and cooperative communication.

Kok Keong Chai received the B.Eng. (Hons.), M.Sc., and Ph.D. degrees from the University of Hertfordshire, Hertfordshire, U.K., in 1998, 1999, and 2007, respectively. He is the Internet of Things Program Lead and Senior Lecturer (Associate Professor) of the Joint Program between Queen Mary University of London (QMUL) and Beijing University of Posts and Telecommunications (BUPT). $\mathrm{He}$ is also a member of the Networks Research Group, QMUL. His current research interests include cooperative multiple-input-single-output transmission schemes, dynamic resource management wireless communications, medium access control for machine-to-machine communications and networks, and sensing and prediction in distributed smart grid networks.

Yue Chen (S'02-M'03-SM'15) B.Eng, M.Eng, Ph.D., MIET, SMIEEE, joined the Networks Research Group, QMUL, in 2000 and continued as a Member of Staff in 2003 after receiving the Ph.D. degree in wireless communications. She is a Professor of telecommunications engineering and QMUL Director of the Joint Program with BUPT. She has been involved in a numerous number of research activities and her research interests focus on intelligent radio resource management for wireless networks, cognitive and cooperative wireless networking, HetNet, smart energy systems, and the Internet of Things. Dr. Chen has served as a Technical Program Committee (TPC) member for many IEEE conferences and is currently an Associate Editor for IEEE COMMUNICATIONS LETTERS.

Jonathan Loo (M'01) received the M.Sc. degree in electronics (with distinction) and the Ph.D. degree in electronics and communications from the University of Hertfordshire, Hertfordshire, U.K., in 1998 and 2003, respectively. Between 2003 and 2010, he was a Lecturer in multimedia communications with the School of Engineering and Design, Brunel University, Uxbridge, U.K. Between June 2010 and May 2017, he was an Associate Professor with the School of Science and Technology, Middlesex University, London, U.K. Since June 2017, he has been a Professor with the School of Computing and Engineering, University of West London, London, U.K, where he leads research in computing and communications engineering. His research interests include information centric networking, cloud computing, networks and protocols, network security, wireless communications, embedded systems, and applied computational intelligent. He has successfully graduated $18 \mathrm{Ph} . \mathrm{D}$. students as their principal supervisor, and has co-authored more than 210 journal and conference papers in the aforementioned specialized areas. Dr. Loo has been an Associate Editor for the Wiley International Journal of Communication Systems since 2011. He was the Lead Editor of the book entitled Mobile Ad Hoc Networks: Current Status and Future Trends (CRC Press, November 2011). 
Shihab Jimaa, an Associate Prof in ECE, has been conducting an international-class research in signal processing including signal analysis, signal classification, and noise and interference cancellation and supervised many projects in the area of adaptive filtering and their applications in signal processing and biomedical engineering where he developed and employed many adaptive algorithms. He has published about 95 research papers in well-known international journals and conferences. Dr. Jimaa is a senior member of the IEEE Communications and Educational Societies, member of the IET, and Fellow member of the higher education academy (HEA)/UK. Dr. Jimaa has extensive international academic experience includes a joint research collaborations with Queen Mary University of London, UK and Korea Advanced Institute of Science and Technology, (Korea). He is the guest editor of the Special Issue on Energy Efficient Wireless Communication Networks with QoS, International Journal of Communication Systems by John Wiley, 2017. Also Dr. Jimaa is an editorial board member of the International journal of Wireless Communication and Simulation.

Youssef Iraqi (S'98-M'03-SM'10) received the M.Sc. and Ph.D. degrees in computer science from the University of Montreal, Canada, in 2000 and 2003, respectively. He is currently an Associate Professor with Khalifa University, United Arab Emirates. He has authored over 100 research papers in international journals and conference proceedings. His research interests include resource management in distributed environments and wireless networking. In 2008, he received the IEEE Communications Society Fred W. Ellersick Prize. 


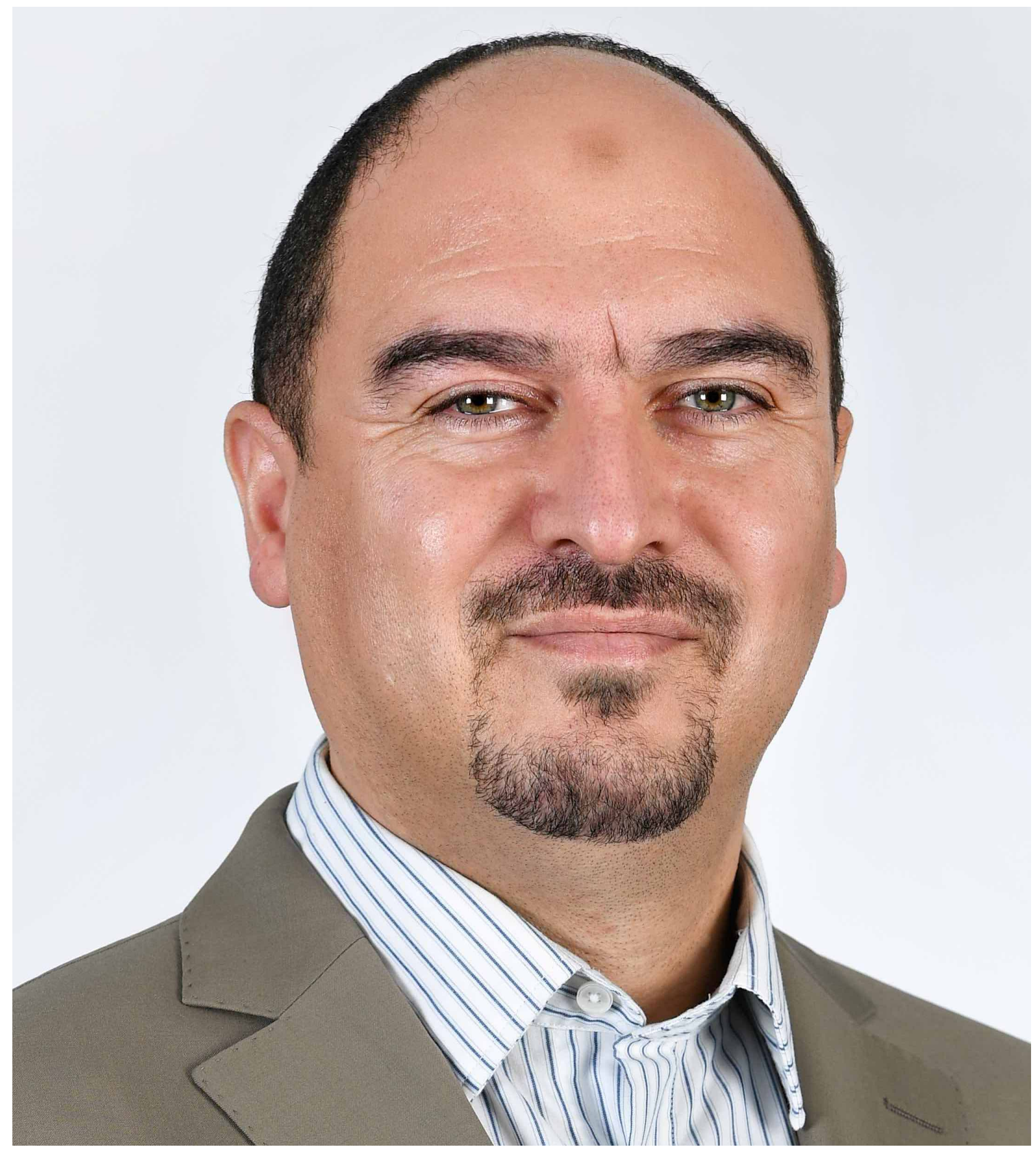

